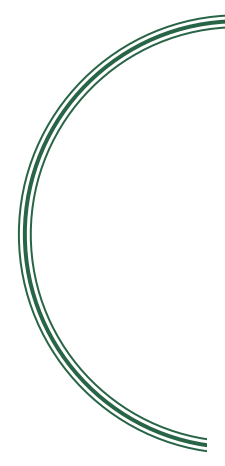

\title{
The Surprising Activities of APOBEC3B and 5-Fluorouracil
}

\author{
Emma L. Walton \\ Staff Writer at the Biomedical Journal, Dronningens gate 56, \\ Trondheim, Norway
}

In this mini-special issue on cancer, we learn how DNA editing enzymes can accelerate the development of cancer and we discover some remarkable effects of the chemotherapeutic agent, 5-fluorouracil, on the immune system. We also discuss a study revealing the continuing problem of vitamin B deficiencies in children in developing countries, and we determine how to distinguish two near-identical forms of necrotizing fasciitis. (Biomed J 2015;38:97-100)

Key words: 5-fluorouracil, APOBEC3, cobalamin, deamination, folate

\section{SPOTLIGHT ON REVIEWS - The Surprising Activities of APOBEC3B and 5-fluorouracil}

$\mathrm{W}$ elcome to this mini-special issue on cancer, where we meet an innate immunity protein with an emerging role in cancer progression and a chemotherapeutic agent with some unexpected properties.

First, Burns et al. ${ }^{[1]}$ introduce us to the apolipoprotein $\mathrm{B}$ mRNA editing enzyme catalytic subunit (APOBEC) family of enzymes that convert cytosine into uracil via a deamination reaction. The largest subset of these genes, the APOBEC 3 family, contains seven members clustered together on chromosome 22 and acts specifically on single-stranded DNA. These proteins play an important role in innate immunity and defend against many viral evaders possessing single-stranded DNA that is mutated and neutralized by deamination. ${ }^{[2]}$ Through the same editing mechanism, APOBEC3 proteins also inhibit the retrotransposition of L1 and human Alu elements ${ }^{[3]}$ and eliminate foreign DNA from cells. ${ }^{[4]}$ Thus, APOBEC 3 proteins are powerful weapons against both endogenous and exogenous viruses.
But could their activity be used against host DNA, akin to the dangers of keeping a loaded gun at home?

The idea that APOBEC proteins are involved in cancer has existed since the discovery of their DNA-mutating properties in 2002. ${ }^{[5]}$ However, catching them red-handed proved difficult because these proteins are highly homologous and most anti-APOBEC antibodies cannot distinguish between them. It was not until 2013 that Harris's team was able to implicate firmly APOBEC3B in the development of cancer by characterizing the mRNA in breast cancer lines. ${ }^{[6]}$ They showed that APOBEC $3 \mathrm{~B}$ is overexpressed in human breast cancer, where it is located in the nucleus and correlates with a high rate of mutation. This work also identified a mutational signature for APOBEC 3B, which was subsequently identified in other cancers. ${ }^{[7]}$ Collectively, these studies suggest that the upregulation of APOBEC3B in developing tumors drives cancer progression [Figure 1].

It is unknown why APOBEC3B is overexpressed in human tumors. Nonetheless, Harris concludes that this protein is likely to be relevant to diagnosis, prognosis, and ultimately therapy, and proposes two distinct therapeutic strategies for manipulating its activity in cancer cells. 


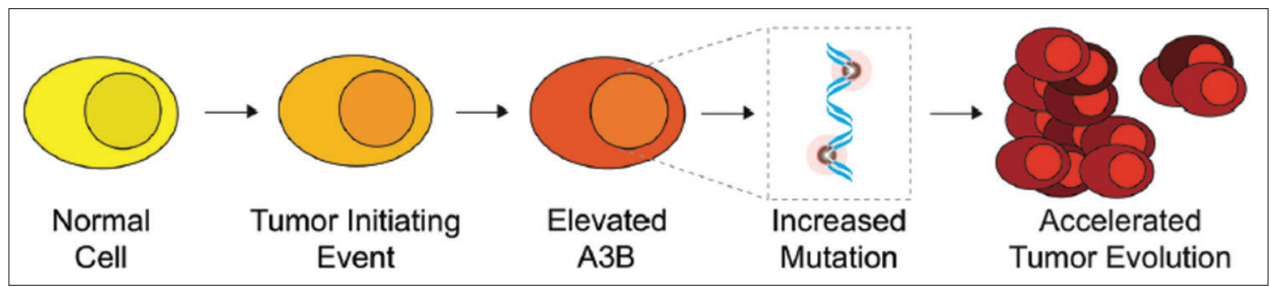

Figure 1: Model describing the contribution of APOBEC3B (A3B) to cancer progression. The upregulation of A3B in developing tumor cells promotes the occurrence of mutations, which speeds up tumor progression. (Figure kindly provided by Prof. R. S. Harris).

Although therapies targeted against APOBEC3B are probably a long way off, the subject of the next review is a therapy that has been used in the clinic for over 50 years, 5-fluorouracil (5-FU). This drug is a pyrimidine analog that competitively blocks the enzymatic activity of thymidylate synthase, limiting the production of thymine nucleotides for DNA synthesis. Thus, 5-FU's mechanism of action is typical of chemotherapeutic drugs that target rapidly dividing cells, which, in turn, inevitably leads to immunosuppression as stocks of white blood cells in the bone marrow are obliterated. However, Ghiringhelli and Apetoh highlight one of the more surprising features of 5-FU: Its ability to stimulate some host immune responses. ${ }^{[8]}$

In cancer patients, the immune system is frequently thwarted by the accumulation of tolerance-promoting regulatory T cells and myeloid-derived suppressor cells (MDSCs) in the blood, lymphoid organs, and in the tumor. Remarkably, however, studies led by Ghiringhelli and Apetoh in mice with thymoma tumors show that 5-FU selectively kills circulating MDSCs by apoptosis without harming B, T, or natural killer (NK) cells. ${ }^{[9]}$ What is more, 5-FU also sensitizes tumors to killing by $\mathrm{CD} 8+\mathrm{T}$ cells by promoting the expression of the Fas receptor on the surface of cancer cells. ${ }^{[10]}$

Nonetheless, the anticancer effect of 5-FU in isolation remains limited, and many studies have assessed its efficacy in combination with other immunomodulatory agents. Take for example, cyclophosphamide, which depletes regulatory $\mathrm{T}$ cells. When used in combination with 5-FU to launch a dual attack on the cellular brakes of the immune system, this approach is highly effective at controlling tumor growth in immunocompetent mice. ${ }^{[9]}$ More combinations are being tested, and will enter Phase II clinical trials this year. Thus, despite its long history, the future still looks bright for 5-FU.

\section{SPOTLIGHT ON ORIGINAL ARTICLES - The Persistent Problem of Vitamin B Deficiencies}

Anemia among young children is a serious problem in developing countries, with an estimated $70 \%$ of children having been affected in India. ${ }^{[11]}$ Deficiency of the essential vitamins folate (vitamin $\mathrm{B}_{9}$ ) or cobalamin (vitamin $\mathrm{B}_{12}$ ) can impair DNA synthesis, leading to the incomplete formation of red blood cells and, subsequently, megaloblastic anemia. In this issue of the Biomedical Journal, Bhadoria and colleagues report the shocking extent of such deficiencies in young children in India. ${ }^{[12]}$

Folate and cobalamin deficiencies occur primarily because of low dietary intake. Folate, as its name suggests (derived from the word "foliage"), is present at high concentrations in green leafy vegetables and in some fruits. Cobalamin, however, can only be obtained from animal products such as meat, fish, and dairy. A recent review by the World Health Organization (WHO) showed that much of the data about the prevalence of these deficiencies is limited to small, local surveys ${ }^{[13]}$ Nonetheless, these studies show that these deficiencies are widespread, occurring in both wealthy and poorer countries. For example, deficiency in cobalamin among individuals over 70 years old in the US population is $\sim 6 \%$, due to the poor absorption of this vitamin in the elderly. ${ }^{[14]}$ Across Latin America, the prevalence of diet-related cobalamin deficiency is around $40 \%,{ }^{[15]}$ whereas in Kenyan school children, it may be as high as $70 \%{ }^{[16]}$

Bhadoria and colleagues focus on a specific subgroup of individuals: Indian children aged between 1 and 5 years. They measured folate and cobalamin levels in 470 children from Delhi and the surrounding region, and obtained information about the diet and socio-demographic status of the children from a questionnaire given to their parents. Overall, the prevalence of cobalamin deficiency was $38 \%$, and low cobalamin levels were associated with belonging to a particular caste and a low standard of living. The prevalence of folate deficiency was $63 \%$ and was surprisingly higher among children with a high standard of living than among those with a low standard of living.

These findings suggest that cobalamin and folate deficiencies are endemic among young children in India, and may contribute to the high prevalence of anemia in this age group. Thus, more effort is required to combat this problem within communities, such as the distribution of cobalamin and folate supplements or conducting food fortification programs.

\section{ALSO IN THIS ISSUE: REVIEWS - Genomic Instability in Chronic Airway Inflammatory Diseases}

Chronic airway inflammatory diseases such as asthma affect millions of people worldwide, but surprisingly little is 
known about their etiology. In this review, ${ }^{[17]}$ Ying and Shen discuss the role of DNA damage response (DDR) in the development of these diseases and the therapeutic potential of specific inhibitors of proteins involved in the DDR pathway.

\section{ORIGINAL ARTICLES - Ban on High Dose Formulations of Gentamicin Justified by Animal Study}

Gentamicin is a valuable antibiotic for the treatment of serious Gram-negative infections, but concerns have been raised about its nephrotoxicity. ${ }^{[18]}$ In this study, Awodele et al. ${ }^{[19]}$ investigate whether the recent ban of high single doses of gentamicin in Nigeria is justified by administering the antibiotic at various doses to rats and assessing subsequent kidney function. Rats treated with high doses of the antibiotic showed significantly elevated creatine levels and acute tubular necrosis, supporting the ban on high-strength gentamicin formulations.

\section{Simple Blood Test Predicts Fetal Outcome in Women with Gestational Diabetes Mellitus}

Between 1 and $14 \%$ of pregnancies are complicated by gestational diabetes mellitus (GDM) ${ }^{[20]}$ In this report, Naha et al..$^{[21]}$ investigate the prognostic value of a glycemic score based on fasting and postprandial glucose levels. They report that glycemic score predicts macrosomia and other congenital abnormalities with high sensitivity and specificity, which has implications for the management of the condition during pregnancy.

\section{Clinical Clues Identify Bacterial Culprits of Necrotizing Fasciitis}

Necrotizing fasciitis is a severe, rapidly progressing soft-tissue infection caused by various types of bacteria, usually occurring in immunocompromised individuals. Hsu et al. ${ }^{[22]}$ retrospectively review 30 diabetic patients in an attempt to define the clinical characteristics to differentiate infections caused by Vibrio vulnificus and those caused by Klebsiella pneumonia, which are indistinguishable in the emergency room. They conclude that exposure history and time between exposure and hospitalization should provide clinicians with important clues as to the origin of infection.

\section{Piercing the Epiphyseal Plate Improves Long-term Outcome of Surgery for Osteogenesis Imperfecta}

Children with the genetic condition osteogenesis imperfecta have brittle bones and are highly susceptible to frac- tures. A radical solution, initially proposed in the 1950s, ${ }^{[23]}$ involves surgically inserting metal rods into the long bones to improve their strength, and has become the mainstay of treatment. Nonetheless, revision surgeries are frequently required. In this study, Li et al. ${ }^{[24]}$ investigate how the exact position of the implant in the femur affects the long-term outcome and report that if the implant penetrates the distal femoral epiphyseal plate, it lasts longer before revision surgery and prolongs survival.

\section{Predicting the Outcome of Unerupted Teeth}

Pericoronal radiolucencies associated with unerupted teeth present a diagnostic dilemma for dentists. Although they usually represent a normal or enlarged dental follicle that requires no intervention, some may signal pathological processes such as a dentigerous cyst. In their retrospective study, Anand et al ${ }^{[25]}$ highlight the extent of this problem and reveal poor correlation between provisional and histological diagnosis of these radiographic anomalies.

\section{Listening to the Airways with an Automated Ear}

Respiratory sounds emanating from the chest wall and mouth are frequently used in the diagnosis of respiratory disorders. However, the ability to interpret these sounds requires a trained, expert ear. Oweis et al. ${ }^{[26]}$ investigate the utility of a computer system for the automated classification of respiratory sounds and report that a system based on artificial neural networks correctly identifies sounds with very high sensitivity and specificity.

\section{PMS is a Neglected Disorder in the Workplace}

Cheng et $a l .{ }^{[27]}$ use a simulated clinical scenario to investigate how young Taiwanese women perceive problems associated with premenstrual syndrome (PMS) in the workplace. Most women neglect the impact of PMS, although in many cases, this condition affects personal relationships, studies, and work, with several cases requiring pharmacological treatment. ${ }^{[28]}$ Thus, more should be done to lift the taboo surrounding PMS in Asian countries and raise awareness of this condition.

\section{BRIEF COMMUNICATION - Surgery is Treatment of Choice for a Rare Complication of Renal Cell Carcinoma}

Chang and colleagues examine how surgery to remove pancreatic metastases influences the outcome of patients 
with renal cell carcinoma. ${ }^{[29]}$ Their retrospective analysis of six cases shows that aggressive surgical management is the best option for tackling this rare complication.

\section{REFERENCES}

1. Burns MB, Leonard B, Harris RS. APOBEC3B: Pathological consequences of an innate immune DNA mutator. Biomed J 2015; 38:102-10.

2. Vieira VC, Soares MA. The role of cytidine deaminases on innate immune responses against human viral infections. BioMed Res Int 2013;2013:683095.

3. Stenglein MD, Harris RS. APOBEC3B and APOBEC3F inhibit L1 retrotransposition by a DNA deamination-independent mechanism. J Biol Chem 2006;281:16837-41.

4. Stenglein MD, Burns MB, Li M, Lengyel J, Harris RS. APOBEC3 proteins mediate the clearance of foreign DNA from human cells. Nat Struct Mol Biol 2010;17:222-9.

5. Harris RS, Petersen-Mahrt SK, Neuberger MS. RNA editing enzyme APOBEC1 and some of its homologs can act as DNA mutators. Mol Cell 2002;10:1247-53.

6. Burns MB, Lackey L, Carpenter MA, Rathore A, Land AM, Leonard B, et al. APOBEC3B is an enzymatic source of mutation in breast cancer. Nature 2013;494:366-70.

7. Rebhandl S, Huemer M, Gassner FJ, Zaborsky N, Hebenstreit D, Catakovic $\mathrm{K}$, et al. APOBEC3 signature mutations in chronic lymphocytic leukemia. Leukemia 2014;28:1929-32.

8. Ghiringhelli F, Apetoh L. Enhancing the anticancer effects of 5-fluorouracil: Current challenges and future perspectives. Biomed J 2015;38:111-6.

9. Vincent J, Mignot G, Chalmin F, Ladoire S, Bruchard M, Chevriaux A, et al. 5-Fluorouracil selectively kills tumor-associated myeloid-derived suppressor cells resulting in enhanced $\mathrm{T}$ cell-dependent antitumor immunity. Cancer Res 2010;70:3052-61.

10. Backus HH, Dukers DF, van Groeningen CJ, Vos W, Bloemena E, Wouters $\mathrm{D}$, et al. 5-Fluorouracil induced Fas upregulation associated with apoptosis in liver metastases of colorectal cancer patients. Ann Oncol 2001;12:209-16.

11. International Institute for Population Sciences (IIPS) and ORC Macro National Family Health Survey (NFHS 3) 2005-2006. Mumbai IIPS. New Delhi: Ajanta Offset and Packagings Limited; 2006. p. 260-90.

12. Kapil U, Toteja GS, Bhadoria AS. Cobalamin and folate deficiencies among children in the age group of 12-59 months in India. Biomed J 2015;38:162-6.

13. Available from: http://www.who.int/nutrition/publications/ micronutrients/FNBvol29N2supjun08.pdf [Last accessed on 2015 Mar 09]
14. Pfeiffer CM, Johnson CL, Jain RB, Yetley EA, Picciano MF, Osterloh JD et al. Trends in blood folate and vitamin B-12 concentrations in the United States, 1988 2004. Am J Clin Nutr 2007;86:718-27.

15. Allen LH. Folate and vitamin B12 status in the Americas. Nutr Rev 2004;62:S29-33.

16. McLean ED, Allen LH, Neumann CG, Peerson JM, Siekmann JH, Demment MW et al. Low plasma vitamin-12 in Kenyan school children is highly prevalent and improved by supplemental animal source foods. J Nutr 2007;13:676-82.

17. Bao Z, Xiong J, Li W, Chen Z, Shen H, Ying S. Genomic instability in chronic airway inflammatory diseases. Biomed J 2015;38:117-24.

18. Humes HD. Aminoglycoside nephrotoxicity. Kidney Int 1988:33:900-11.

19. Awodele O, Tomoye OP, Quashie NB, Amagon KI, Ogunnowo SA. Gentamicin nephrotoxicity: Animal experimental correlate with human pharmacovigilance outcome. Biomed J 2015;38:125-30.

20. American Diabetes Association. Gestational diabetes mellitus. Diabetes Care 2003;26 Suppl 1:S103-5.

21. Naha K, Naha S, Pandit VR, Seshadri S. A simple score to predict fetal outcomes in gestational diabetes mellitus. Biomed J 2015; 38:131-5.

22. Hsu JC, Shen SH, Yang TY, Chen PH, Huang KC, Tsai YH Necrotizing fasciitis and sepsis caused by Vibrio vulnificus and Klebsiella pneumoniae in diabetic patients. Biomed $\mathrm{J}$ 2015;38:136-42.

23. Sofield HA ME. Fragmentation, realignment, and intramedullary rod fi xation of deformities of the long bones in children: A ten- year appraisal. J Bone Joint Surg Am 1959;41:1371-91.

24. Li WC, Kao HK, Yang WE, Chang CJ, Chang CH. Femoral non-elongating rodding in osteogenesis imperfecta - The importance of purchasing epiphyseal plate. Biomed J 2015;38:143-7.

25. Anand S, Kashyap B, Kumar GR, Shruthi BS, Supriya AN. Pericoronal radiolucencies with significant pathology: Clinico-histopathologic evaluation. Biomed J 2015;38:148-52.

26. Oweis RJ, Abdulhay EW, Khayal A, Awad A. An alternative respiratory sounds classification system utilizing artificial neural networks. Biomed J 2015;38:153-61.

27. Cheng SH, Sun ZJ, Lee IH, Shih CC, Chen KC, Yang YK et al. Perception of premenstrual syndrome and attitude of evaluations of work performance among incoming university female students. Biomed J 2015;38:167-72.

28. Dimmock PW, Wyatt KM, Jones PW, O'Brien PM. Efficacy of selective serotonin-reuptake inhibitors in premenstrual syndrome: A systematic review. Lancet 2000;356:1131-6.

29. Chang YH, Liaw CC, Chuang CK. The role of surgery in renal cel carcinoma with pancreatic metastasis. Biomed J 2015;38:173-6. 